Cytokine stimulus

\title{
Modulation of inflammatory response arising from high-intensity intermittent and concurrent strength training in physically active males
}

\author{
Paula Alves Monteiro ${ }^{\mathrm{a}, \mathrm{b}, *}$, Eduardo Zapaterra Campos ${ }^{\mathrm{a}, \mathrm{f}}$, Flaviane Poleto de Oliveira ${ }^{\mathrm{a}}$, \\ Fernando Pierin Peres ${ }^{c}$, José Cesar Rosa-Neto ${ }^{\mathrm{d}}$, Gustavo Duarte Pimentel ${ }^{\mathrm{e}}$, Fabio Santos Lira ${ }^{\mathrm{a}, *}$ \\ ${ }^{a}$ Exercise and Immunometabolism Research Group, Department of Physical Education, Universidade Estadual Paulista, Presidente Prudente, São Paulo, Brazil \\ ${ }^{\mathrm{b}}$ Center and Prescription Motor Activity Laboratory, Department of Physical Education, Universidade Estadual Paulista, Presidente Prudente, São Paulo, Brazil \\ ${ }^{\mathrm{c}}$ Universidade do Oeste Paulista, São Paulo, Brazil \\ ${ }^{\mathrm{d}}$ Immunometabolism Research Group, Institute of Biomedical Sciences, University of São Paulo (USP), São Paulo, SP, Brazil \\ e Laboratório de Investigação em Nutrição Clínica e Esportiva (Labince), Faculdade de Nutrição (FANUT), Universidade Federal de Goiás, Goiânia, Brazil \\ ${ }^{\mathrm{f}}$ Department of Physical Education, Federal University of Pernambuco, Brazil
}

\section{A R T I C L E I N F O}

\section{Article history:}

Received 29 July 2016

Received in revised form 27 November 2016

Accepted 9 December 2016

Available online 30 December 2016

\section{Keywords:}

HIIT

Concurrent strength

Inflammation

Immune status

\begin{abstract}
A B S T R A C T
The purposes of this study were to determine: (i) the extent of an acute session of high-intensity intermittent exercise (HIIE) followed by a concurrent strength session (Conc) on the increase of systemic inflammatory cytokines and chemokines, and (ii) whether eight weeks of high intensity interval training plus concurrent strength training alters the acute inflammatory response and immune status. Ten recreationally active males (aged $26.9 \pm 4.3$ years) performed two experimental exercise sessions interspersed by eight weeks of HIIT plus concurrent strength training. The experimental exercise session was composed of a $5-\mathrm{km}$ run on a treadmill (1:1 at $100 \%$ of maximal aerobic speed (MAS)), and after 10 min of passive recovery, back squat exercises were performed ( $80 \% 1 \mathrm{RM}$, four sets until exhaustion). Serum samples were collected after fasting, pre-HIIE, post-HIIE, Pre-Conc, Post-Conc, and 30 and 60 min postexercise session. The comparison between both concurrent exercise sessions was performed using repeated measure ANOVA, with the Bonferroni Post-hoc when necessary. Interleukin-6 (IL-6) presented a moment effect $(F=6.72 ; p<0.05)$, with Post-Conc significantly higher than pre-HIIE, Post-HIIE, and $60 \mathrm{~min}$, only a tendency was found between pre-HIIE and post-HIIE (difference $=-5.99 ; \mathrm{p}=0.09$ ). MCP-1 and IL-1ra did not present effects for condition, moment, or interaction. Interleukin-10 (IL-10) presented both moment and interaction effects $(F=5.31$ and 2.50; $p=0.005$ and 0.036$)$. Pre-Conc and Post-Conc were significantly higher than Pre-HIIE. The interaction between before and after eight weeks of concurrent training probably occurred at Post-Conc $\left(11.42 \pm 3.09 \mathrm{pg} \mathrm{mL}^{-1}\right.$ and $\left.8.88 \pm 1.29 \mathrm{pg} \mathrm{mL}^{-1}\right)$. In addition, maintenance of immune function was observed. Therefore, HIIE and concurrent strength exercise lead to an increase in cytokines response, but eight weeks of training program promoted antiinflammatory response after an acute session of concurrent exercise.
\end{abstract}

(c) 2016 Elsevier Ltd. All rights reserved.

\section{Introduction}

Exercise controls several functions of the human body, and recently, the discovery of the skeletal muscle as a metabolically active organ has further increased the need to study the effects of training protocols on the production of myokines [1]. Myokines are molecules produced by the skeletal muscle due to contraction, have different functions in the organism, and are capable of acting either locally or in other tissues [2].

\footnotetext{
* Corresponding authors at: Departamento de Educação Física, Universidade Estadual Paulista (UNESP), Presidente Prudente, São Paulo, Brazil (P.A. Monteiro).

E-mail address: paulinha_1003@hotmail.com (P.A. Monteiro).
}

Some myokines, such as tumor necrosis factor (TNF $\alpha$ ), interleukins 6 and 1 beta (IL-6 and 1 $\beta$ ) were found in higher concentrations after strenuous exercise when compared to baseline [3]. In addition, these myokines have been studied in order to investigate the acute anti-inflammatory effects of exercise [4]. Likewise, IL-6 and interleukin-10 (IL-10) play an anti-inflammatory role and have been observed in increased amounts in response to exercise [5-9]. Therefore, exercise is able to modulate inflammatory disorders associated with high levels of myokine and glycoprotein expression in skeletal muscle tissue, leading to changes in immune cells, i.e., reduction in toll-like receptor expression on the cell surface of monocytes and increased frequency of regulatory $\mathrm{T}$ cells $[1,10]$. 
These acute changes seem to be able to transiently generate systemic elevation of anti-inflammatory cytokines (mainly IL-10 and interleukin 1 receptor antagonist - IL-1ra), regardless of the modulation of pro-inflammatory cytokines $[11,12]$.

Monocyte chemoattractant protein 1 (MCP-1) is also known to be an important chemokine, and it is responsible for recruitment of monocytes. Thus, targeting MCP-1 may prevent the changes associated with macrophage-induced inflammation [13]. Recent evidence suggest that the adaptive response to exercise is facilitated by a boost of the inflammatory response, mediated through the increase of the non-resident macrophage pool in reaction to a robust elevation in MCP-1 [14]. Interestingly, these events are reported to coincide with a significant reduction in delayed onset of muscle soreness [14], suggesting that the increase of monocyte response via MCP-1 signaling may lead to an enhanced recovery.

Typically, endurance training mediates anti-inflammatory actions that lead to fat loss and improvement of aerobic capacity. However, research recently demonstrated that high-intensity intermittent exercise cause anti-inflammatory responses similarly to moderate-intensity continuous exercise [7-9]. In addition, we have demonstrated that strength exercise and concurrent strength training promote changes in inflammatory response [15-17].

Zwetsloot et al. [9] showed that two weeks of a high intensity interval training (HIT) program did not alter the inflammatory response after an acute high intensity exercise (HIIE) session. We recently demonstrated that a 5-km HIIE session is able to increase levels of IL-6 and IL-10, similarly to steady state exercise. Lately, concurrent training (i.e., aerobic + strength training in the same session) has been proposed to induce metabolic adaptations [18]. However, Navalta et al. [19] observed that repeated intense interval exercise appears to affect the immunological system, leading to an immunosuppression status by reducing the potential vulnerability to antigens during this timeframe.

Although widely used in the literature [7,8,20-23], little is known about the acute inflammatory response to HIIE followed by concurrent strength exercises, and the effects of concurrent training on inflammatory response. Indeed, understanding the immunometabolic effects of HIIE plus strength training is crucial for clinical practice, once it gains more followers around the world. Therefore, the aim of study was to analyze the inflammatory response of concurrent exercise (HIIE + strength training) after eight weeks of training among physically active subjects.|

\section{Methods}

\subsection{Subjects}

The sample consisted of 10 males aged between 18 and 35 $(26.9 \pm 4.3)$ years old (Table 1$)$. The participants were considered physically active according to aerobic conditioning determined

\section{Table 1}

Mean and standard deviation of IL-6, IL-10, MCP-1, and IL-1ra at the 1st week and after eight weeks of concurrent training $(n=10)$.

\begin{tabular}{llll}
\hline Variables & $\begin{array}{l}\text { Pre-training } \\
(1 \text { st week) }\end{array}$ & $\begin{array}{l}\text { Post-training } \\
\text { (8th week) }\end{array}$ & p-value \\
\hline Weight $(\mathrm{kg})$ & $74.13(7.8)$ & $74.97(8.2)$ & 0.121 \\
$\mathrm{VO}_{2 \max }$ & $53.60(3.6)$ & $54.52(3.8)$ & 0.000 \\
1RM & $113.50(22.9)$ & $135.20(24.4)$ & 0.001 \\
Inflammatory markers & & & \\
IL-6 $\left(\mathrm{pg} \mathrm{mL}^{-1}\right)$ & $10.94 \pm 6.27$ & $9.79 \pm 5.08$ & 0.60 \\
IL-10 $\left(\mathrm{pg} \mathrm{mL}^{-1}\right)$ & $8.08 \pm 1.59$ & $8.27 \pm 1.56$ & 0.78 \\
$\mathrm{MCP}-1\left(\mathrm{pg} \mathrm{mL}^{-1}\right)$ & $137.87 \pm 29.90$ & $128.51 \pm 20.81$ & 0.80 \\
IL-1ra $\left(\mathrm{pg} \mathrm{mL}^{-1}\right)$ & $716.67 \pm 454.65$ & $763.85 \pm 469.66$ & 0.86 \\
\hline
\end{tabular}

by $\mathrm{VO}_{2 \text { Peak }}$ and presented no characteristics of any clinical problem that could prevent physical activity. The subjects participated voluntarily in the study after being informed of the procedures, risks, and benefits, and signed an consent form. This study was approved by the Ethics Committee (22793414.7.0000.5402).

\subsection{Experimental design}

In order to analyze acute inflammatory response before and after concurrent training, the participants were submitted to a concurrent exercise session (Pre-training), followed by eight weeks of concurrent training, and another concurrent exercise session (Posttraining). Prior to the Pre-training period, participants were familiarized with the half-squat exercise. After adaptation, they were submitted to (i) one incremental exercise test in order to determine maximal aerobic speed (MAS) and, (ii) one maximal repetition of half-squat with guided bar (1-RM). At least $48 \mathrm{~h}$ after the tests, the subjects performed both Pre-training and Post-training. Between the tests the subjects trained twice a week for eight weeks (Fig. 1).

\subsection{Incremental test}

To determine aerobic fitness, the participants performed a maximal incremental test on the treadmill (Inbramed-ATL) until exhaustion, with the measurement of maximum oxygen consumption (Model Quark PFT Ergo - Cosmed - Rome) $\left(\dot{\mathrm{VO}}_{2}\right)$. Each stage of the test lasted two minutes; the first stage was performed at $8 \mathrm{~km} \mathrm{~h}^{-1}$, increasing by $1 \mathrm{~km} \mathrm{~h}^{-1}$ at the end of each stage. In addition, heart rate was monitored using a heart rate monitor (Polar Vantage NV, Electro Oy, Finland) integrated into the gas analysis system. The average of the final $30 \mathrm{~s}$ was defined as peak oxygen uptake $\left(\mathrm{V}_{2 \text { peak }}\right)$. The maximal speed reached in the test was defined as the peak speed $\left(S_{\text {peak }}\right)$. When the participant was not able to finish the two minutes stage, the speed was expressed according to the permanence time in the final stage, determined as the following equation: $S_{\text {peak }}=$ speed of penultimate stage + [(time, in seconds, remained in the final stage multiplied by $\left.\left.1 \mathrm{~km} \mathrm{~h}^{-1}\right) / 120 \mathrm{~s}\right]$ [24].

\subsection{Maximum dynamic strength test}

Seventy-two hours after the maximum endurance running test, the participants performed the maximum dynamic strength test. It consisted of the maximum load that the individual was able to lift in just one-repetition maximum (1RM) in the half squat exercise with a guided bar. The recommendations of the American Society of Exercise Physiology were used for this test [25].

Before performing the $1 \mathrm{RM}$ test, participants were familiarized with the half squat exercise, during which they executed the medium squat twice for 15-20 repetitions, the first with no weight and the second with 10-15 kg weight, for three days, once a day, for adaptation to the machine and for the correct execution of the movement.

Subsequently, the 1RM test was performed after a five minutes warm-up at $50 \%$ of vVO2max. Next, subjects performed eight repetitions at the estimated intensity of 50\% 1RM, followed by another three repetitions at $80 \%$ estimated 1 RM. Attempts to establish the 1 RM were only executed with progressively heavier weight until fatigue. The resting interval was three to five minutes and the number of attempts was no more than five. The highest weight lifted in the test was regarded as the value of 1RM.

For better control of the 1RM test procedures, body position and feet placement of each participant in the half-squat exercise was recorded and reproduced throughout the study. In addition, a woo- 


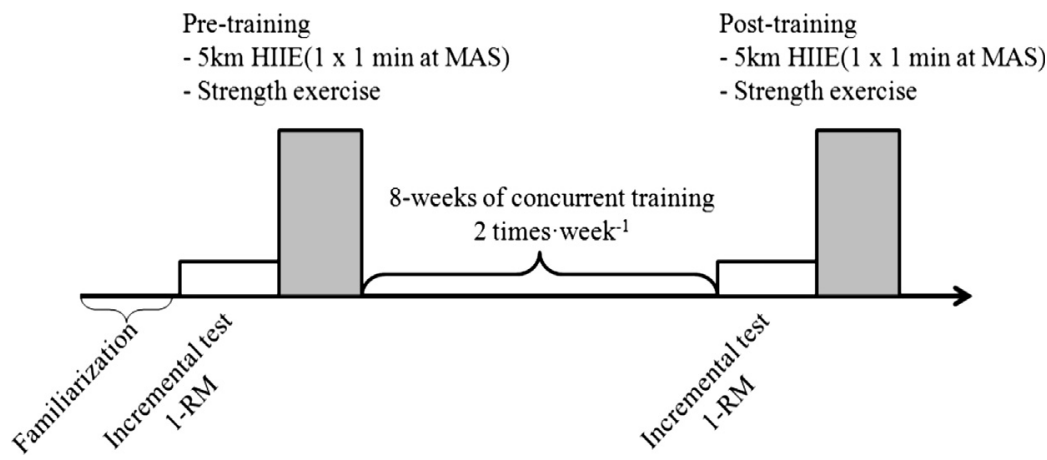

Fig. 1. Experimental design of acute concurrent exercise session and effects of 8-weeks of concurrent training on inflammatory response.

den seat with adjustable height was placed behind the participant in order to keep the bar displacement and knee angle $\left(\sim 90^{\circ}\right)$ constant in each half-squat repetition.

\subsection{Pre- and post-assessment}

Pre and post assessments were performed in the morning, at baseline and after eight weeks of training, and were composed of blood samples, breakfast, and a session of HIIT and strength exercises (the same as the training).

\subsection{Immune system evaluation}

An upper respiratory tract infection questionnaire, adapted from Bassit et al. [26], was used to assess the participants' immune status. It is known that high intensity physical activity promotes light to moderate immune suppression, affecting the subject's health and performance. The questionnaire consisted of a list of symptoms or infections that were marked by the subjects during the period of the study.

\subsubsection{Blood samples}

Blood samples were collected during the following periods: (1) overnight fast (eight to $12 \mathrm{~h}$ ); (2) prior to training session; (3) immediately after training session; (4) 30 min after training session; (5) 60 min after training session.

\subsubsection{Breakfast}

After the fasting blood sample, a standard breakfast was offered (consisting of toast, yogurt, and cottage cheese) with an energy intake value stipulated by body composition, comprising energy values distributed between carbohydrates, proteins, lipids, and fiber.

\subsubsection{Training program - HIIT plus strength training}

The subjects performed a warm-up consisting of running on a treadmill at $50 \%$ of $S_{\text {peak }}$ for five minutes at a $1 \%$ inclination. The HIIT was performed intermittently with subjects running on the treadmill for one minute at $100 \%$ of $S_{\text {peak }}$, interspersed by one minute of passive recovery (without exercise) until they had completed $5-\mathrm{km}$. Participants performed three sets, $10 \mathrm{~min}$ apart, with a 90 s resting interval between series, with a load of 8-12 repetition maximum (RM) in the half-squat, bench press, knee extension, back handle, knee flexion, elbow flexion (curl), ankle flexion, and elbow extension in the pulley. The number of repetitions was maintained between 8 and 12RM, and when participants achieved more than $12 \mathrm{RM}$, the load was adjusted to remain in the planned intensity zone (Fig. 2).

\subsection{Blood analyses}

Approximately $20 \mathrm{~mL}$ of blood was collected in tubes containing EDTA and anti-coagulant gel to separate serum and plasma. The blood was centrifuged at $3000 \mathrm{rpm}$ for $15 \mathrm{~min}$ at $4{ }^{\circ} \mathrm{C}$. Next, the aliquots of serum and plasma were stored in plastic tubes, Eppendorf type, and stored at $-20^{\circ} \mathrm{C}$ for further analysis. The concentration of MCP-1 and IL-1ra were analyzed through enzymatic assay by ELISA using kits from RD System (Minneapolis, USA). The concentration of IL-10 and IL- 6 were analyzed through enzymatic assay by ELISA using kits from eBioscience, Ambriex S/A, (São Paulo-SP, Brazil).

\subsection{Statistical analysis}

Descriptive data were shown as means and standard deviation. In addition, the paired Student $\mathrm{T}$ test was used for comparison of the variables between the pre-training and post-training. Twoway analysis of variance (ANOVA) repeated measures, with Bonferroni's post hoc analysis, was applied to compare changes in inflammatory response and immune status after eight weeks of training (condition [pre-training and post-training]), moment (pre-HIIE, post-HIIE, pre-Conc, post-Conc, $30 \mathrm{~min}$ and $60 \mathrm{~min}$ ) and interaction (moment $\times$ condition). The statistical significance was set at $5 \%$ and all analyses were conducted using SPSS, version 17.0 (SPSS Inc. Chicago. IL).

\section{Results}

The baseline values of IL-6, IL-10, MCP-1, and IL-1ra before and after eight weeks of concurrent training presented no differences between values from the 1 st to the 8 th week (Table 1 ).

The IL-6 did not present any condition effect $(F=0.001$; $\mathrm{p}=0.97)$ or interaction effect $(\mathrm{F}=0.859 ; \mathrm{p}=0.458)$, but we found moment effect $(F=4.36 ; p=0.013)$. IL-10 also presented a moment effect $(F=5.31 ; p=0.005)$, but did not show interaction effect (condition vs. moment) $(\mathrm{F}=2.50 ; \mathrm{p}=0.083)$ or condition effect $(\mathrm{F}=0.63 ; \mathrm{p}=0.43)$. MCP-1 and IL-1 ra did not present effects for condition, moment, or interaction (Table 2). As shown in Figs. 1 and 2 , it is possible to identify where is the difference in the moments for IL-6 and IL- 10 .

Immune system activity was assessed by the number of marks made on the questionnaire. Each mark represented a symptom or infection observed by the participant, therefore, the lower number of marks meant better immune system function. No difference was observed between pre-training and post-training $(p>0.05)$, demonstrating the maintenance of the immune function. Figs. 3 and 4. 
TRAINING PROGRAM

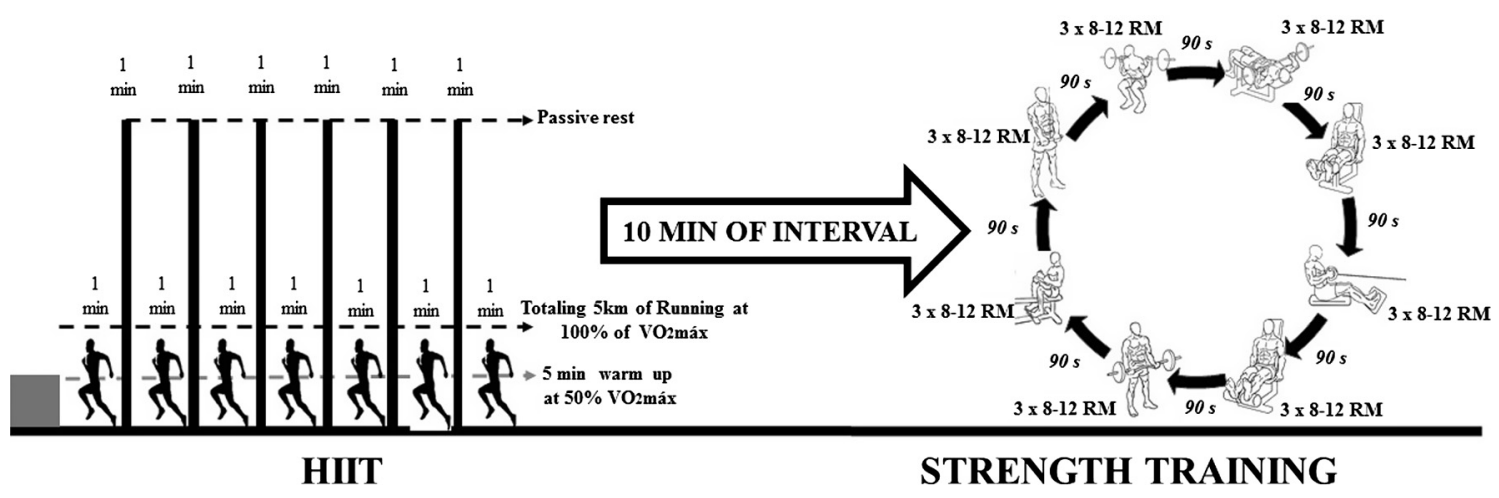

Fig. 2. Training program.

Table 2

Effect of moment, condition and interaction (moment vs condition) during pre (Pre-Training $=1$ st week) and post (Post-Training $=8$ th week) concurrent training.

\begin{tabular}{|c|c|c|c|c|c|c|c|}
\hline & & $\begin{array}{l}\text { Pre training } \\
\Delta \text { Mean }(\mathrm{SD})\end{array}$ & $\begin{array}{l}\text { Post training } \\
\Delta \text { Mean }(\mathrm{SD})\end{array}$ & Effect & $\mathrm{F}$ & p-value & Effect size \\
\hline \multirow[t]{7}{*}{ IL-6 $\left(\mathrm{pg} \mathrm{mL}^{-1}\right)$} & Pre and Post HIIE & $6.1(7.8)$ & $1.9(12.5)$ & & & & \\
\hline & Pre and post Conc & $4.9(5.1)$ & $3.2(4.4)$ & & & & \\
\hline & Pre HIIE and post Conc & $10.5(10.8)$ & $6.4(12.6)$ & Condition & 0.001 & 0.977 & 0.000 \\
\hline & Pre HIIE and $30 \mathrm{~min}$ & $2.3(6.8)$ & $5.0(14.6)$ & Moment & 4.361 & 0.013 & 0.238 \\
\hline & Pre HIIE and $60 \mathrm{~min}$ & $1.8(3.0)$ & $-2.6(10.4)$ & Moment $\times$ condition & 0.859 & 0.458 & 0.058 \\
\hline & Post Conc and $30 \mathrm{~min}$ & $-8.2(13.1)$ & $-1.3(6.1)$ & & & & \\
\hline & Post Conc and $60 \mathrm{~min}$ & $-8.7(11.2)$ & $-9.0(6.5)$ & & & & \\
\hline \multirow[t]{7}{*}{$\mathrm{IL}-10\left(\mathrm{pg} \mathrm{mL}^{-1}\right)$} & Pre and post HIIE & $1.0(1.2)$ & $0.6(1.6)$ & & & & \\
\hline & Pre and post Conc & $1.9(2.7)$ & $-1.0(2.2)$ & & & & \\
\hline & Pre HIIE and post Conc & $3.3(2.6)$ & $0.8(2.1)$ & Condition & 0.633 & 0.437 & 0.034 \\
\hline & Pre HIIE and $30 \mathrm{~min}$ & $1.3(2.3)$ & $0.9(1.3)$ & Moment & 5.311 & 0.005 & 0.228 \\
\hline & Pre HIIE and $60 \mathrm{~min}$ & $0.3(0.5)$ & $0.4(1.0)$ & Moment $\times$ condition & 2.503 & 0.083 & 0.122 \\
\hline & Post Conc and $30 \mathrm{~min}$ & $-2.0(3.9)$ & $0.1(3.2)$ & & & & \\
\hline & Post Conc and $60 \mathrm{~min}$ & $-3.0(2.5)$ & $-0.4(2.3)$ & & & & \\
\hline \multirow[t]{7}{*}{$\mathrm{MCP}-1\left(\mathrm{pg} \mathrm{mL}^{-1}\right)$} & Pre and post HIIE & $38.4(48.8)$ & $22.8(33.2)$ & & & & \\
\hline & Pre and post Conc & $5.2(99.3)$ & $26.9(22.4)$ & & & & \\
\hline & Pre HIIE and post Conc & $31.6(87.2)$ & $39.3(54.8)$ & Condition & 1.382 & 0.261 & 0.096 \\
\hline & Pre HIIE and $30 \mathrm{~min}$ & $3.6(31.4)$ & $25.6(34.7)$ & Moment & 0.807 & 0.385 & 0.058 \\
\hline & Pre HIIE and $60 \mathrm{~min}$ & $7.4(46.4)$ & $24.8(54.2)$ & Moment $\times$ condition & 0.011 & 0.917 & 0.001 \\
\hline & Post Conc and $30 \mathrm{~min}$ & $-15.8(71.6)$ & $1.1(18.7)$ & & & & \\
\hline & Post Conc and $60 \mathrm{~min}$ & $-24.2(56.5)$ & $-14.4(19.4)$ & & & & \\
\hline \multirow[t]{7}{*}{ IL-1 ra (pg mL $\left.{ }^{-1}\right)$} & Pre and post HIIE & $102.4(376.5)$ & $34.7(38.2)$ & & & & \\
\hline & Pre and post Conc & $75.9(436.1)$ & $143.4(324.2)$ & & & & \\
\hline & Pre HIIE and post Conc & $-5.2(150.1)$ & $18.2(372.7)$ & Condition & 0.617 & 0.452 & 0.064 \\
\hline & Pre HIIE and $30 \mathrm{~min}$ & $-1.2(120.6)$ & $69.8(359.2)$ & Moment & 0.852 & 0.410 & 0.087 \\
\hline & Pre HIIE and 60 min & $-92.9(282.5)$ & $-29.3(159.9)$ & Moment $\times$ condition & 0.497 & 0.553 & 0.052 \\
\hline & Post Conc and $30 \mathrm{~min}$ & $728.5(566.6)$ & $565.2(532.9)$ & & & & \\
\hline & Post Conc and $60 \mathrm{~min}$ & $-120.9(377.3)$ & $-172.7(251.2)$ & & & & \\
\hline
\end{tabular}

Notes: bold values $=$ significant difference with $\mathrm{p}<0.05$.

\section{Discussion}

The present study aimed to investigate the acute inflammatory response of an HIIE followed by a concurrent strength exercise, as well as the effects of eight weeks of concurrent training on inflammatory status. The main findings of the present study were that: (i) during pre-training and post-training, HIIE associated with a concurrent strength exercise was able to increase IL- 6 and IL-10 concentrations, while no changes occurred in MCP-1 or IL1ra levels, and (ii) probably due to the eight weeks of concurrent training, a similar acute concurrent exercise caused lower peak of IL-10 concentration at post-training when compared to pre-training.

Understanding the acute and transient inflammatory response to an exercise bout may be useful for training prescription, and it could also be important to investigate the effects of exercise on acute inflammatory response and immune function [9,27]. Likewise, inflammatory response has been commonly investigated in different types of exercises (cycling, running, strength), intensities (HIIE, steady state exercise, low-intensity exercise, all-out bout) and volumes (low to high). However, the present study sought to explain the inflammatory response in association with both concurrent exercise and the chronic effects of concurrent training.

In the present study, we identified that IL- 6 concentrations increased in a similar manner after eight weeks of concurrent training. Moreover, it was observed that at the post-training moment, IL-6 levels remained low up to the post-Conc session, leading to speculation that after concurrent training, a different inflammatory response occurs after HIIE or concurrent strength exercise. 


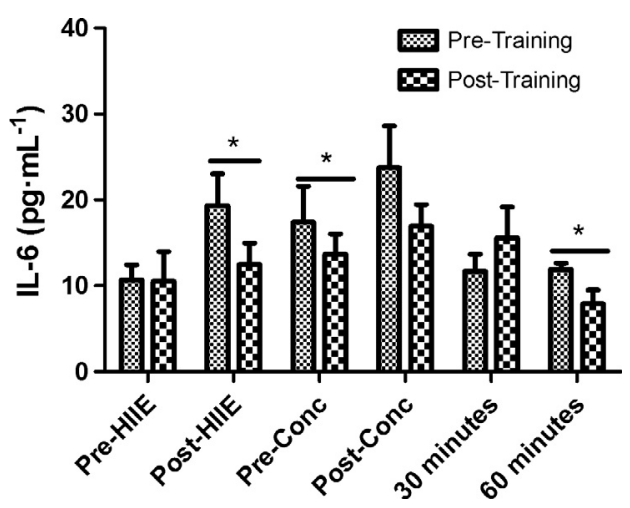

Fig. 3. IL-6 concentration before, during, and after the acute high intensity interval exercise and concurrent strength exercise pre (Pre-Training $=1$ st week) and post (Post-Training $=8$ th week) concurrent training. *Significantly different from PostConc. $\mathrm{n}=10$.

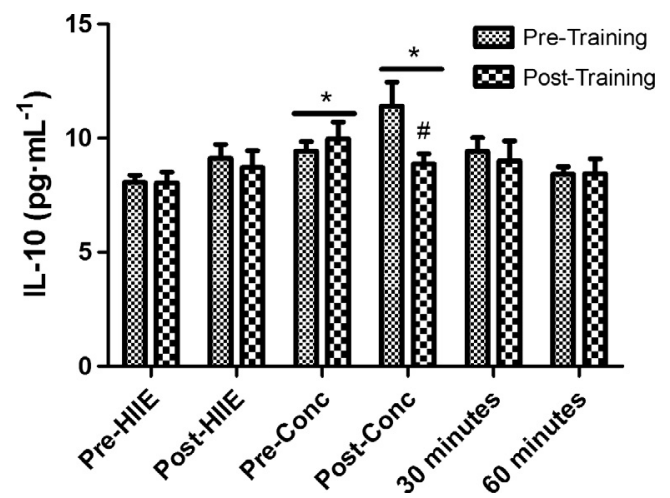

Fig. 4. IL-10 concentration before, during and after the acute high intensity interval exercise and concurrent strength exercise pre (Pre-Training $=1$ st week) and post (Post-Training $=8$ th week) concurrent training. *Significantly different of Pre-HIIE; ${ }^{\#}$ significantly different of Post-Conc Pre-Training. $\mathrm{n}=10$.

In a previous study, our group demonstrated that a 5-km HIIE session increased IL-6 ( 150\%) concentrations immediately after exercise, while continuous exercise presented a divergent increase in IL-6 ( $\sim 50 \%$ ) concentrations; however, we did not use concurrent strength exercise [7]. Likewise, Inoue et al. [15] observed an increase in IL-6 levels after a similar protocol. Higher IL-6 values can favor the gluconeogenesis process and availability of glucose for skeletal muscle. These results suggest that alterations regarding cytokine kinetics during exercise are dependent on the type of exercise. In addition, little is known about the alterations in IL-6 levels after concurrent training. Zwetsloot et al. [9] found that two weeks of HIIT did not alter IL-6 concentrations after an HIIE session. Although, the study used a shorter training period and lower intensity than ours, IL-6 cytokine level after training was similar when compared to the present study.

Additionally, we observed an increase in IL-10 concentrations immediately after the concurrent strength exercise. However, this result disagrees with Zwetsloot et al. [9], who showed increased IL10 levels only $45 \mathrm{~min}$ after exercise. Nevertheless, the lower volume and absence of concurrent strength exercise may explain the difference between studies. Increased IL-10 after different exercise types indicates that acute bouts of exercise induce an anti-inflammatory status, which may explain the positive adaptations induced by training. Although IL-10 is considered an antiinflammatory cytokine, which remains increased after exercise [7-9], we observed that at post-training, peak IL-10 levels were lower than at pre-training. On the other hand, this result contra- dicts the Zwetsloot et al. [9] study, which did not observe modifications in IL-10 concentrations after 2-weeks of HIIT. In addition, the reduction in IL-10 peak after training may indicate an adaptation in the immune system to concurrent training [28].

Furthermore, we did not find any effect on IL1 ra or MCP-1 after pre-training or post-training exercise. However, Zwetsloot et al. [9] found an increase in MCP-1 levels after HIIE without any adaptation to HIIT. Moreover, the cytokine levels after acute concurrent exercise pre and post concurrent training also remained unchanged.

Additionally, we evaluated immune system activity during the training period and found that moderate intensity exercise is related mainly with improvement in immune status instead of the negative influence caused by high intensity exercise or overtraining. Furthermore, although the immune system status is controlled by nutritional conditions and overall health, it is influenced by high intensity exercises $[28,29]$. Indeed, the exacerbation of the anti-inflammatory profile observed in HIIT could lead to immunosuppression, as found in highly trained athletes [27,28], however our results did not show HIIT-induced impaired immune response either from the acute or chronic exercise interventions.

The first limitation of the present study is the absence of a control group performing isolated HIIT or strength training. Secondly, since the subjects were all physically active males, it would be interesting to investigate whether this response is similar for obese, diabetic or elderly subjects. Finally, the assessment of overall immune activity by questionnaire can also be considered a limitation.

In summary, we identified that both IL-6 and IL-10 increased after concurrent exercise (HIIE plus strength exercise) and the immune system seemed to adapt after concurrent training because IL-10 concentrations were lower at post-training than pre-training, indicating that the immune system was restored after a training program when compared to single exercise bout. Therefore, our data suggest that in clinical practice, the eight weeks of exercise program (short-term) promotes beneficial inflammatory response, such as, adequate macrophages recruitment and immune function.

\section{Disclosure statement}

No competing financial interests exist.

\section{Acknowledgments}

The authors would like to thank FAPESP for their support (2013/25310-2).

\section{References}

[1] B.K. Pedersen, Muscular interleukin-6 and its role as an energy sensor, Med. Sci. Sports Exerc. 44 (2012) 392-396.

[2] B.K. Pedersen, M.A. Febbraio, Muscle as an endocrine organ: focus on musclederived interleukin-6, Physiol. Rev. 88 (2008) 1379-1406.

[3] K. Ostrowski, T. Rohde, S. Asp, P. Schjerling, B.K. Pedersen, Pro- and antiinflammatory cytokine balance in strenuous exercise in humans, J. Physiol. 15 (1999) 287-291.

[4] C. Brandt, B.K. Pedersen, The role of exercise-induced myokines in muscle homeostasis and the defense against chronic disease, J. Biomed. Biotechnol. (2010) Article ID e520258.

[5] B.K. Pedersen, Special feature for the Olympics: effects of exercise on the immune system: exercise and cytokines, Immunol. Cell Biol. 78 (2000) 532535.

[6] B.K. Pedersen, M.A. Febbraio, Muscles, exercise and obesity: skeletal muscle as a secretory organ, Nat. Rev. Endocrinol. 8 (2012) 457-465.

[7] C. Cabral-Santos, J. Gerosa-Neto, D.S. Inoue, V.L. Panissa, L.A. Gobbo, A.M. Zagatto, E.Z. Campos, F.S. Lira, Similar anti-inflammatory acute responses from moderate intensity continuous and high-intensity intermittent exercise, J. Sports Sci. Med. 14 (2015) 849-856

[8] F.S. Lira, V.L. Panissa, U.F. Julio, E. Franchini, Differences in metabolic and inflammatory responses in lower and upper body high-intensity intermittent exercise, Eur. J. Appl. Physiol. 115 (2015) 1467-1474 
[9] K.A. Zwetsloot, C.S. John, M.M. Lawrence, R.A. Battista, R.A. Shanely, Highintensity interval training induces a modest systemic inflammatory response in active, young men, J. Inflamm. Res. 9 (2014) 9-17.

[10] M. Gleeson, N. Bishop, D. Stensel, et al., The anti-inflammatory effects of exercise: mechanisms and implications for the prevention and treatment of disease, Nat. Rev. Immunol. 11 (2011) 607-615.

[11] A. Steensberg, C.P. Fischer, C. Keller, et al., IL-6 enhances plasma IL-1ra, IL-10, and cortisol in humans, Am. J. Physiol. Endocrinol. Metab. 285 (2003) E433E437.

[12] R.Q. Landers-Ramos, N.T. Jenkins, E.E. Spangenburg, et al., Circulating angiogenic and inflammatory cytokine responses to acute aerobic exercise in trained and sedentary young men, Eur. J. Appl. Physiol. 114 (2014) 1377-1384

[13] T.L. Cranford, R.T. Enos, K.T. Velázquez, J.L. McClellan, J.M. Davis, U.P. Singh, M. Nagarkatti, P.S. Nagarkatti, C.M. Robinson, E.A. Murphy, Role of MCP-1 on inflammatory processes and metabolic dysfunction following high-fat feedings in the FVB/N strain, Int. J. Obes. 40 (2016) 844-851.

[14] M.R. Deyhle, A.M. Gier, K.C. Evans, D.L. Eggett, W.B. Nelson, A.C. Parcell, R.D. Hyldahl, Skeletal muscle inflammation following repeated bouts of lengthening contractions in humans, Front. Physiol. 6 (2016) 424.

[15] D.S. Inoue, V.L. Panissa, P. Monteiro, J. Gerosa Neto, F.E. Rossi, B.M. Antunes, E. Franchini, J.M. Cholewa, L.A. Gobbo, F.S. Lira, Immunometabolic responses to concurrent training: the effects of exercise order in recreational weightlifters, J. Strength Cond. Res. 30 (2015) 1960-1967.

[16] F.E. Rossi, J. Gerosa-Neto, N.E. Zanchi, J.M. Cholewa, F.S. Lira, Impact of short and moderate restintervals on the acute immunometabolic response to exhaustive strength exercise, J. Strength Cond. Res. 30 (2015) 1563-1569.

[17] J. Gerosa-Neto, F.E. Rossi, E.Z. Campos, B.M. Antunes, J.M. Cholewa, F.S. Lira Impact of short and moderate rest intervals on the acute immunometabolic response to exhaustive strength exercise: part II, J. Strength Cond. Res. 30 (2016) 1570-1576.

[18] D. Docherty, B. Sporer, A proposed model for examining the interference phenomenon between concurrent aerobic and strength training, Sports Med 30 (2000) 385-394.
[19] J.W. Navalta, R.A. Tibana, E.A. Fedor, A. Vieira, J. Prestes, Three consecutive days of interval runs to exhaustion affects lymphocyte subset apoptosis and migration, Biomed. Res. Int. (2014) 694801.

[20] G. Tschakert, J. Kroepfl, A. Mueller, O. Moser, W. Groeschl, P. Hofmann, How to regulate the acute physiological response to "aerobic" high-intensity interval exercise, J. Sports Sci. Med. 14 (2015) 29-36.

[21] O. Moser, G. Tschakert, A. Mueller, W. Groeschl, T.R. Pieber, B. ObermayerPietsch, G. Koehler, P. Hofmann, Effects of high-intensity interval exercise versus moderate continuous exercise on glucose homeostasis and hormone response in patients with type 1 diabetes mellitus using novel ultra-longacting insulin, PLoS ONE 10 (2015) e0136489.

[22] C. Foster, C.V. Farland, F. Guidotti, M. Harbin, B. Roberts, J. Schuette, A. Tuuri, S T. Doberstein, J.P. Porcari, The effects of high intensity interval training vs steady state training on aerobic and anaerobic capacity, J. Sports Sci. Med. 14 (2015) 747-755.

[23] K.M. Wood, B. Olive, K. LaValle, H. Thompson, K. Greer, T.A. Astorino, Dissimilar physiological and perceptual responses between sprint interval training and high-intensity interval training, J. Strength Cond. Res. 30 (2016) 244-250.

[24] H. Kuiper, FT, Verstappen, H.A. Keizer, P. Geurten, G. Van Kranenburg, Variability of aerobic performance in the laboratory and its physiologic correlates, Int. J. Sports Med. 6 (1985) 197-201.

[25] L.E. Brown, J.P. Weir, ASEP procedures recommendation I: accurate assessment of muscular strength and power, J. Exerc. Physiol. 4 (2001) 1-21.

[26] R.A. Bassit, L.A. Sawada, R.F. Bacurau, F. Navarro, L.F. Costa Rosa, The effect of BCAA supplementation upon the immune response of triathletes, Med. Sci. Sports Exerc. 32 (2000) 1214-1219.

[27] J.C. Neto, F.S. Lira, M.T. de Mello, R.V. Santos, Importance of exercise immunology in health promotion, Amino Acids 41 (2011) 1165-1172.

[28] D.C. Nieman, Exercise immunology: practical applications, Int. J. Sports Med. 18 (1997) S91-S100.

[29] L.T. Mackinnon, Immunity in athletes, Int. J. Sports Med. 18 (1997) S62-S68. 